\title{
Neurodevelopmental masks of rolandic spectrum disorders (clinics, diagnostic and management).
}

Zaytsev's Psychoneurological Center Itd. Ligivsky prospect, 50 liter $F$

Russian Federation, Saint-Petersburg

Dmitry Zaytsev, Ilya Zaytsev - psychiatry

Nikolay Titov - neurology

Introduction. According to modern views, there is a spectrum of atypical variants of the age-related childhood epilepsy with centro-temporal spikes in the EEG (former rolandic epilepsy). This spectrum includes atypical age-related childhood epilepsy with centro-temporal spikes in the EEG accompanied by motor and/or mental disorders. It is also possible to identify a large number of neurological and psychopathological conditions (attention deficit, speech delay, sluttering, enuresis, encopresis etc.), accompanied by certain changes in the EEG without epileptic seizures.
Aim. To identify correlation between neurodevelopmental disorders with specific alterations in the EEG and its influence on management tactics.

North-Western State Medical University named after I.I.Mechnikov.

Kirochnaya st, 41

Russian Federation, Saint-Petersburg Alexandr Sofronov, llya Zaytsev - psychiatry

Material and methods. We analyzed the pool of case histories of patients with age-related childhood disorder, violation of attention and with speech delay. The age of the patients was between 3 and 14 years. Patients with disorders in the form of enuresis, encopresis and stuttering were excluded from the study because of the small number. Seizures were also exclusion criteria.

Group I: 42 children aged 6-14 years with an established diagnosis of ADHD.

Group II: 40 children aged 3-12 years with speech delay. All children were examined by a child psychiatrist and neurologist at least 2 times for 6 months, a speech therapist in dynamics. To all patients in both groups were performed a long-term EEG monitoring with a night sleep record (duration of at least 12 hours) at least twice in the observation period.
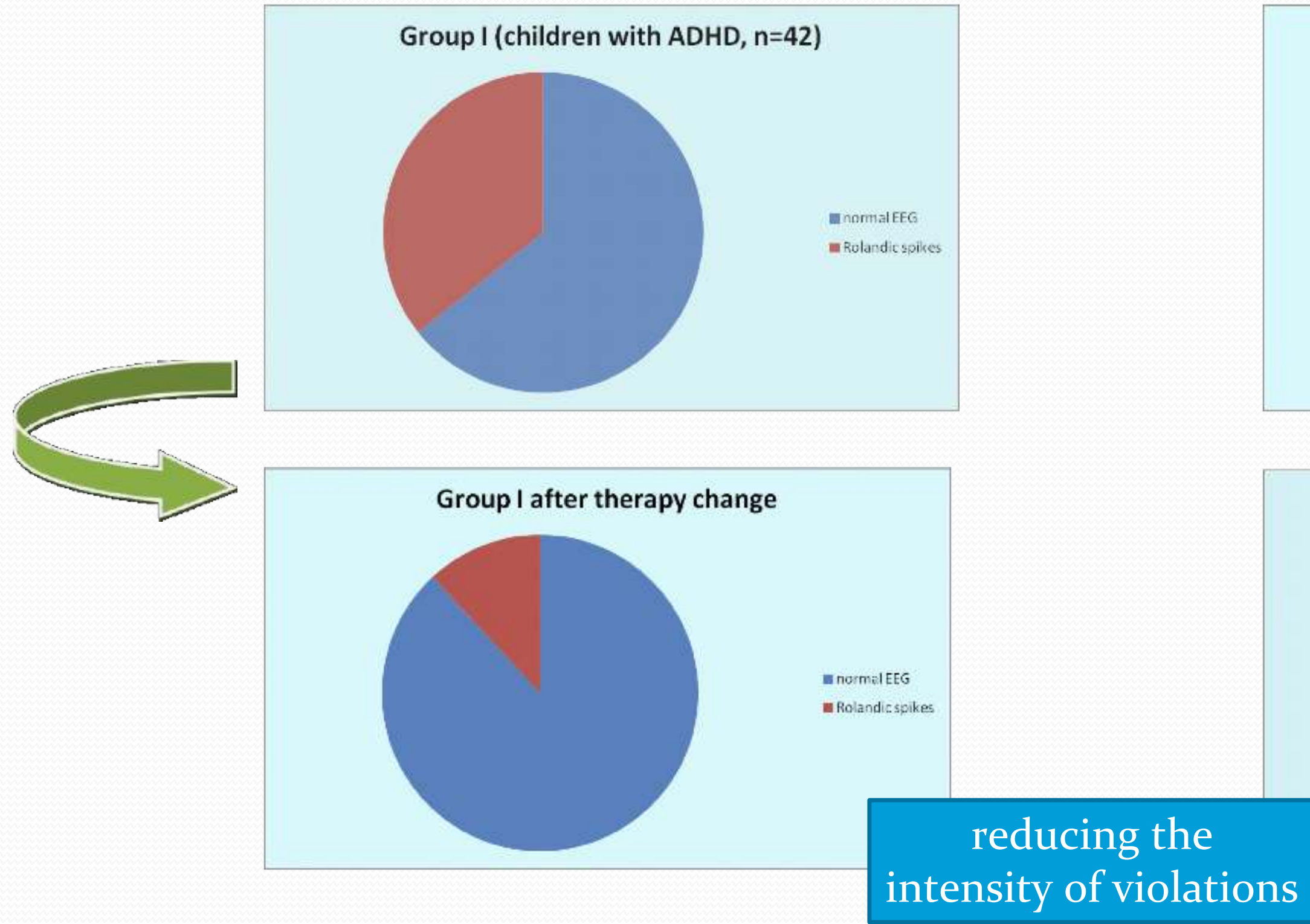
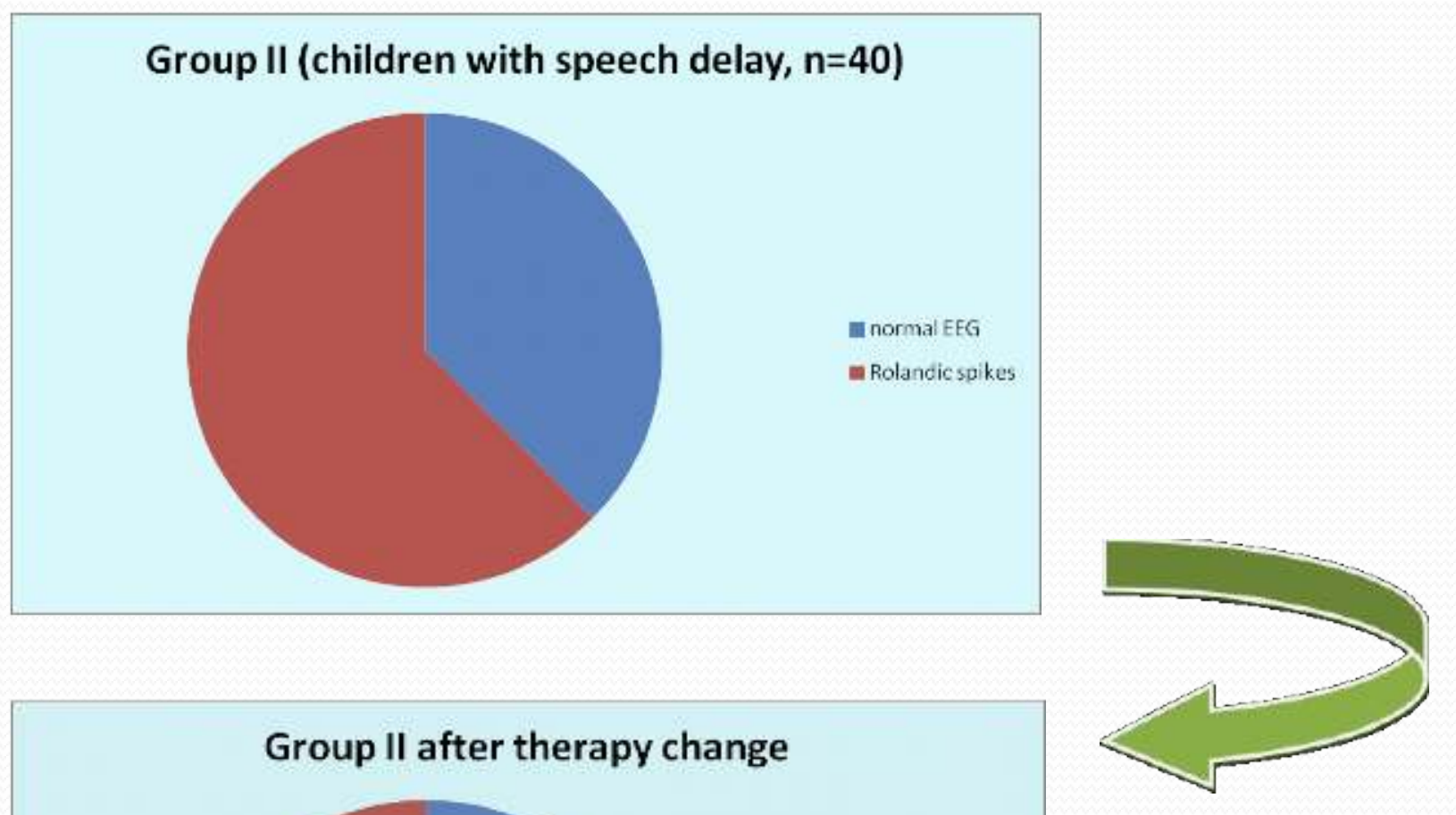

Results. 15 of 42 (35\%) of patients in group I and 25 of $40(62.5 \%)$ patients in group II had EEG disorders in the form of rolandic spikes. The detection of such disorders led to a change in the treatment tactics in the form antiepileptic drugs addition, which led to a significant improvement of speech skills (group II) and reducing the intensity of attention disorders (group I) in all patients, who had EEG improvement regardless of leading nosology.

\section{Conclusion.}

1.Disturbances in the EEG are a full-fledged but not subsidiary symptom in the diagnosis and choice of tactics for treating disorders such as ADHD and speech delay in children.

2.There is a clear relationship between the disorders in the neurological and psychiatric status and the violations in the EEG in the form of rolandic spikes. 